\title{
Correction: Loss of ZNF32 augments the regeneration of nervous lateral line system through negative regulation of SOX2 transcription
}

\author{
Yuyan Wei ${ }^{1}$, Kai Li ${ }^{1}$, Shaohua Yao ${ }^{2}$, Junping Gao ${ }^{1}$, Jun Li ${ }^{1}$, Yanna Shang ${ }^{3}$, Jie Zhang ${ }^{1}$, \\ Le Zhang ${ }^{1}$, Yanyan $\mathrm{Li}^{1}$, Xianming $\mathrm{Mo}^{3}$, Wentong Meng ${ }^{3}$, Rong Xiang ${ }^{5}$, Jiankun Hu${ }^{4}$, \\ Ping Lin ${ }^{1}$, Yuquan Wei ${ }^{2}$ \\ ${ }^{1}$ Division of Experimental Oncology, State Key Laboratory of Biotherapy, West China Hospital, Sichuan University, and \\ Collaborative Innovation Center for Biotherapy, Chengdu, P.R. China \\ ${ }^{2}$ Division of Cancer Biotherapy, State Key Laboratory of Biotherapy, West China Hospital, Sichuan University, and Collaborative \\ Innovation Center for Biotherapy, Chengdu, P.R. China \\ ${ }^{3}$ Laboratory of Stem Cell Biology, State Key Laboratory of Biotherapy, West China Hospital, Sichuan University, and \\ Collaborative Innovation Center for Biotherapy, Chengdu, P.R. China \\ ${ }^{4}$ Department of Gastrointestinal Surgery and Laboratory of Gastric Cancer, State Key Laboratory of Biotherapy, West China \\ Hospital, Sichuan University, and Collaborative Innovation Center for Biotherapy, Chengdu, P.R. China \\ ${ }^{5}$ Department of clinical medicine, School of Medicine, Nankai University and Collaborative Innovation Center for Biotherapy, \\ Tianjin, P.R. China
}

Published: December 24, 2019

Copyright: Wei et al. This is an open-access article distributed under the terms of the Creative Commons Attribution License 3.0 (CC BY 3.0), which permits unrestricted use, distribution, and reproduction in any medium, provided the original author and source are credited.

This article has been corrected: In Figure 6F, the images of P-1-169 group from Figure 5D were accidentally duplicated. The corrected Figure 6 is shown below. The authors declare that these corrections do not change the results or conclusions of this paper.

Original article: Oncotarget. 2016; 7:70420-70436. https://doi.org/10.18632/oncotarget.11895 


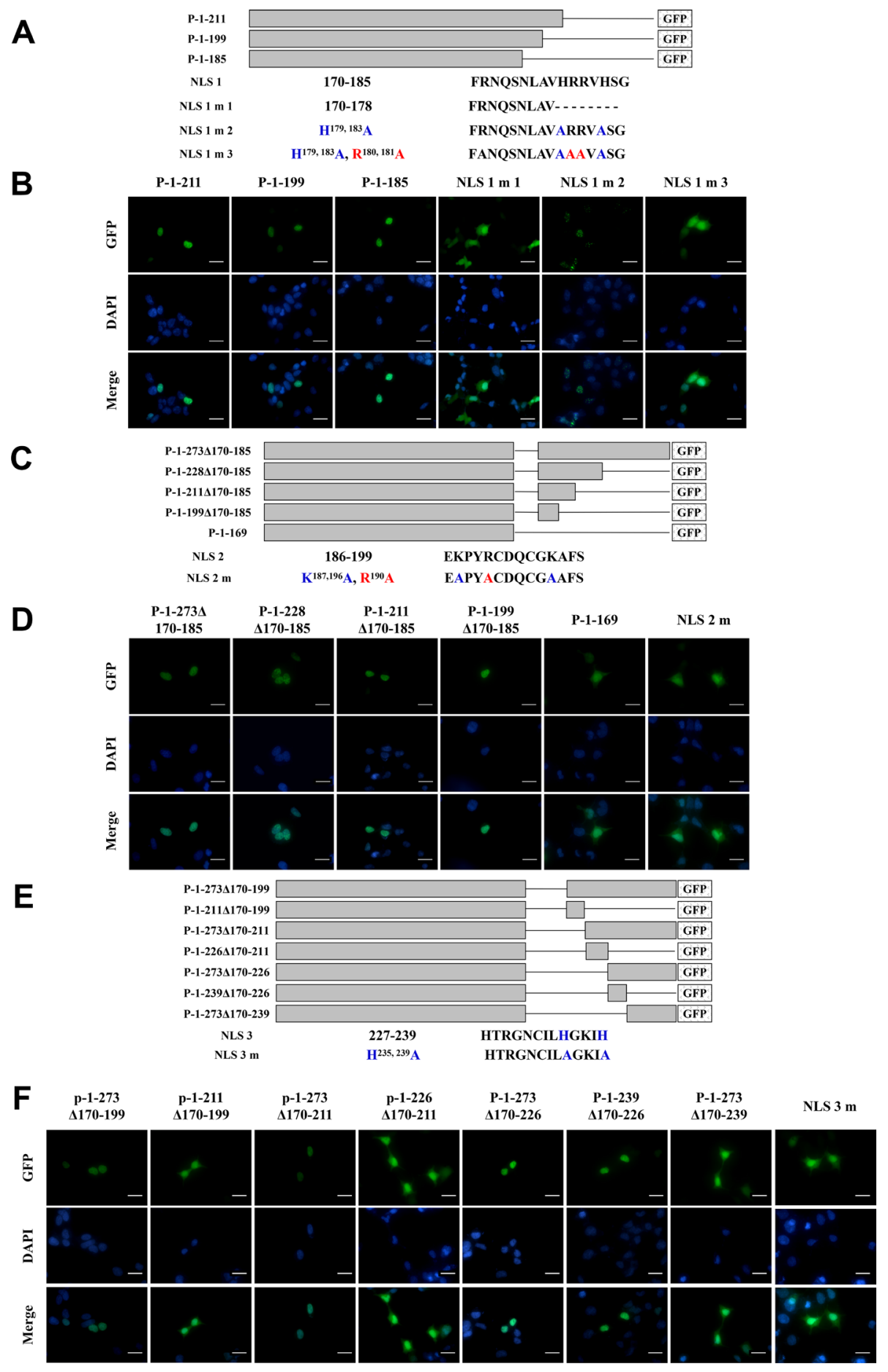

Figure 6: Identification of NLSs in ZNF32 and the localization of NLS mutants. (A, B) ZNF32 NLS 1 (Aa 170-185). (A) Schematic representation of recombinant, GFP-tagged ZNF32 mutant proteins. Lines represent the deleted sequences in the proteins. (B) The subcellular localization of ZNF32 and NLS 1 mutant proteins. Recombinant proteins are shown in green (GFP), and cell nuclei are shown in blue (DAPI). (C, D) ZNF32 NLS 2 (Aa 186-199). The basic amino acids Lys and Arg were replaced with Ala in NLS 2 mutant. (C) Schematic representation of the recombinant, GFP-tagged ZNF32 mutants. Lines represent the deleted sequences in the proteins. (D) The subcellular localization of ZNF32 and NLS 2 mutant. Recombinant proteins are shown in green (GFP), and cell nuclei are shown in blue (DAPI). (E, F) ZNF32 NLS 3 (Aa 227-239). (E) Schematic representation of the recombinant, GFP-tagged ZNF32 mutants. Lines represent the deleted sequences in the proteins. (F) The subcellular localization of ZNF32 and NLS 3 mutant. Recombinant proteins are shown in green (GFP), and cell nuclei are shown in blue (DAPI). Scale bar $=50 \mu \mathrm{m}$. 